«SABAR, SAMA THIOSANOU » (SABAR, MA TRADITION). FRONTIËRES ET PROPRIÉTÉ CULTURELLES DANS LA TRANSMISSION DES DANSES SÉNÉGALAISES EN EUROPE

\author{
Alice Aterianus-Owanga
}

Université de Poitiers | «Revue européenne des migrations internationales »

2019/3 Vol. 35 | pages 153 à 174

ISSN 0765-0752

Article disponible en ligne à l'adresse :

https://www.cairn.info/revue-europeenne-des-migrations-

internationales-2019-3-page-153.htm

Distribution électronique Cairn.info pour Université de Poitiers.

(C) Université de Poitiers. Tous droits réservés pour tous pays.

La reproduction ou représentation de cet article, notamment par photocopie, n'est autorisée que dans les limites des conditions générales d'utilisation du site ou, le cas échéant, des conditions générales de la licence souscrite par votre établissement. Toute autre reproduction ou représentation, en tout ou partie, sous quelque forme et de quelque manière que ce soit, est interdite sauf accord préalable et écrit de l'éditeur, en dehors des cas prévus par la législation en vigueur en France. Il est précisé que son stockage dans une base de données est également interdit. 
«Sabar, sama thiosanou » (sabar, ma tradition). Frontières et propriété culturelles dans la transmission des danses sénégalaises en Europe

"Sabar, sama thiosanou" (Sabar, my Tradition). Cultural Boundaries and Property in the Transmission of Senegalese Dances in Europe «Sabar, sama thiosanou» (sabar, mi tradición). Fronteras y propiedad culturales en la transmisión de los bailes senegaleses en Europa

\section{Alice Aterianus-Owanga}

\section{(2) OpenEdition}

\section{Journals}

Édition électronique

URL : http://journals.openedition.org/remi/13737

DOI : $10.4000 /$ remi. 13737

ISSN : $1777-5418$

Éditeur

Université de Poitiers

Édition imprimée

Date de publication : 1 décembre 2019

Pagination : 153-174

ISBN : 979-10-90426-65-8

ISSN : 0765-0752

Distribution électronique Cairn

CAIRN

CHERCHER, REPÉRER, AVANCER.

Référence électronique

Alice Aterianus-Owanga, « «Sabar, sama thiosanou » (sabar, ma tradition). Frontières et propriété culturelles dans la transmission des danses sénégalaises en Europe », Revue européenne des migrations internationales [En ligne], vol. 35 - n³ et 4 | 2019, mis en ligne le 01 janvier 2022, consulté le 20 avril 2020. URL : http://journals.openedition.org/remi/13737; DOI : https://doi.org/10.4000/remi. 13737 


\section{"Sabar, sama thiosanou " (sabar, ma tradition). Frontières et propriété culturelles dans la transmission des danses sénégalaises en Europe}

\section{Alice Aterianus-Owanga ${ }^{1}$}

"Salam aleykoum la famille, bonjour tout le monde, al hamdoulilah je remercie le bon Dieu, mes parents. Je suis né fils de griot de père et mère, sabar ma culture qui résonne dans mes oreilles [suit une série d'émoticones en forme de cœurs rouges]. Depuis tout petit cet instrument que j'admire beaucoup et que j'aime du fond du cœur, qui me permet de partager ma culture avec des gens qui aime notre culture sénégalais qui est la danse sabar. Je vous dis encore merci, c'était magnifique ; merci les musiciens, merci les élèves et à bientôt inch'Allah ; sabar, sama thiosanou [ma tradition]. " ${ }^{2}$

Maf Sing Sing, musicien et danseur sénégalais installé dans le Sud de la France, publiait ce témoignage sur sa page Facebook, en octobre 2018, au sortir d'un stage de danse sabar, organisé à Marseille, durant lequel il avait enseigné ses savoirs chorégraphiques à des passionnés de danses africaines. Présenté ici comme sa " culture " et sa " tradition ", le sabar est un instrument de musique, un événement festif et un répertoire de danses, associés initialement à l'ethnie ${ }^{3}$ wolof et lébou du Sénégal et à la caste ${ }^{4}$ des griots. Parfois discrédité au Sénégal pour la transgression des normes de genre qui y est associée, le sabar s'est diffusé depuis les années 1970 hors des frontières du Sénégal et du continent africain pour devenir un emblème de la culture sénégalaise. Cette diffusion transnationale est survenue par le canal d'artistes en migration qui, comme Maf Sing Sing, enseignent le sabar en Occident, ou par la médiation des musiciens de mbalax qui, à l'instar de Youssou N'Dour, l'ont fait connaître sur les scènes de musiques du monde. Comme dans cet exemple, le sabar est conçu par de

1 Maitre Assistante Ambizione, ISSR, Université de Lausanne, Bâtiment Anthropole 5088, Quartier Dorigny, 1015 Lausanne, Suisse ; aliceaterianus@yahoo.fr

2 Extrait de la page Facebook du musicien Maf Sing Sing (page publique). J'ai conservé l'orthographe original, tout en ajoutant des éléments de ponctuation afin de clarifier la lecture.

3 Au contraire d'une idée d'unité culturelle fixe et homogène, la notion d'ethnie est employée ici en référence à une construction historique forgée largement durant la période coloniale (Amselle et M'Bokolo, 1985) et objet d'usages stratégiques dans les sociétés contemporaines.

4 Patricia Tang et d'autres auteurs traitant de la question des castes au Sénégal analysent comment la notion de griot recoupe des appartenances multiples et mouvantes, relevant du lignage, de la famille, de l'ethnicité ou de l'idée de communauté (Tang, 2007: 53). 
nombreux artistes ayant migré en Europe comme un moyen de " communication interculturelle " : tout en étant identifiée comme un patrimoine national sénégalais, cette danse pourrait être partagée auprès des Occidentaux et permettrait de transcender les frontières culturelles entre Sénégalais et Européens ${ }^{5}$.

Différentes études anthropologiques ont examiné la manière dont les pratiques musicales et dansées accompagnaient la fabrique, la représentation et l'incorporation d'une nation imaginée (Askew, 2001 ; Hugues-Freeland, 2008). Depuis quelques années, des recherches réfléchissent aussi au rôle que la musique et la danse exercent dans la recomposition de ces communautés nationales dans un contexte de migration (Averill, 1994 ; Bohlman, 2011 ; Salzbrunn, 2017 ; Turino et Lea, 2004). Parallèlement, d'autres travaux examinent comment les musiques et les danses voyagent pour être appropriées par d'autres groupes sociaux, comme dans le cas de la salsa, du tango ou du hip-hop, désormais " globalisés " et resignifiés en différents endroits (Apprill, 2017 ; Djebbari, dans ce dossier ; Mitchell, 2001). La diffusion du sabar se situe à I'intersection de ces différents phénomènes : d'une part, il est devenu le moyen de construire une " communauté émotionnelle " éphémère lors des rencontres de la diaspora (Aterianus-Owanga, 2018) ; de l'autre, il fait I'objet de diverses adaptations afin d'être transmis auprès de nouveaux publics, en Europe ${ }^{6}$ ou aux Amériques (Bizas, 2014). Toutefois, à la différence de pratiques globalisées qui se rattachent à des berceaux multiples et dont les ancrages relèvent davantage de la logique du rhizome que de la racine, le cas du sabar offre un exemple original de forme musicale et dansée dont la diffusion transnationale n'a pas empêché qu'elle demeure ancrée dans un territoire, une idée de localité et une revendication nationaliste.

Cet article se base sur l'observation de différents espaces d'enseignement du sabar en Europe (cf. Encadré 1), afin d'examiner comment la transmission de cette performance s'accompagne d'un jeu sur les frontières, (re)définissant tantôt les contours de la nation sénégalaise, tantôt les distinctions raciales entre Africains et Occidentaux. Ma démarche s'inscrit dans la continuité d'une tradition de recherche socio-anthropologique qui a imposé la notion de frontières comme catégorie d'analyse propice à la compréhension de la dimension relationnelle des identités et appartenances (ethniques, nationales, raciales, etc.) (Barth, 1995 ; Jeanpierre, 2010 ; Lamont et Molnár, 2002). S'éloignant de I'acception de la frontière comme territoire, Fredrik Barth initia cette approche en affirmant qu'au-delà d'une idée de traits culturels partagés, les groupes sociaux et les identités culturelles sont le résultat d'une dynamique constante d'établissement, de maintien et de transformation des frontières, par " des processus sociaux d'exclusion et d'incorporation " que l'ethnologue doit s'attacher à examiner (Barth, 1995 : 204-205). Dans sa continuité, une série de recherches anthropologiques se sont saisies de cet outil pour contester les perspectives substantialistes des identités culturelles, éclairant leur dimension relationnelle et situationnelle. Dans cet article, j'emprunte cette théorie afin de m'intéresser au

5 Les identifications comme "Occidentaux ", "Africains ", " Noirs ", " Blancs " renvoient dans cet article à des catégories émiques résultant de constructions sociohistoriques et non à des catégories d'analyse.

6 Les artistes de sabar sont majoritairement installés dans des pays d'Europe de l'Ouest, mais aussi dans les pays scandinaves et, dans une moindre mesure, en Europe de l'Est. 
rôle de certaines ressources symboliques - les performances de sabar - dans la création, le maintien ou la contestation des différences entre des groupes sociaux et des appartenances qu'ils revendiquent. La notion de frontières me permet d'englober différents types de distinctions identitaires opérées au travers des pratiques musicales et dansées (dans le domaine de l'ethnicité, de la nation ou de la race) et de mettre en exergue leur articulation avec les rapports de pouvoir (Lamont et al., 2001). Je considère ainsi les sites d'enseignement du sabar comme des espaces d'observation de la production relationnelle des frontières et des appartenances, et j'interroge la façon dont dans un contexte de migration, la confrontation à l'autre renforce et reconfigure l'affirmation d'une " communauté imaginée " (Anderson, 1996).

Après une première partie retraçant la construction du sabar comme tradition nationale au Sénégal, une seconde partie de ce texte se penche sur les modalités d'implantation de cette danse en Europe. Je m'intéresse aux pratiques, discours et pédagogies au travers desquels quelques enseignants de sabar, en jouant sur la tension entre tradition et individuation qui caractérise cette danse, ont converti ce répertoire national en pratique appropriable par différents individus/ groupes sociaux. Puis j'examinerai les différents ressorts au travers desquels le sabar continue à servir l'affirmation d'une idée de localité, d'identité et de frontière nationale, tout en se transnationalisant. Entrainant des circulations spatiales et des conversions identitaires des élèves européennes, le sabar représente le véhicule d'un apparent dépassement des frontières culturelles et un moyen d'inclusion de l'altérité. Cependant, l'examen des résistances et polémiques qui traversent ce champ artistique soulignera que sous certains aspects, et notamment sur le plan économique, l'enseignement du sabar s'accompagne d'une affirmation de frontières exclusives, révélant des inégalités et des rapports de force. La dissolution ou l'affirmation des frontières par la pratique du sabar se module alors de façon variée en fonction des rapports de pouvoir qui traversent ce monde artistique en migration, et que les artistes redessinent via leurs pratiques.

\section{Encadré 1 : Méthodologie}

\footnotetext{
Cette recherche est basée sur une ethnographie intensive et multisituée des réseaux de diffusion du sabar, menée depuis février 2017 entre différentes villes du continent européen et du Sénégal. Ma recherche inclut des entretiens semi-directifs (cinquante), une participation observante des espaces de performance et d'enseignement et un suivi de trajectoires de circulation entre le Sénégal et l'Europe. Bien que j'aie effectué quelques entretiens avec des acteurs rencontrés à une seule reprise, je me suis attachée à entretenir des relations régulières avec une partie de mes interlocuteurs. Pour dépasser les résistances initiales - liées aux asymétries décrites dans cet article et dont je suis partie prenante en tant que femme blanche européenne -, j'ai négocié mon immersion en tâchant de m'impliquer dans des activités profitables aux artistes (prise de photos et de vidéos) et en développant des liens de confiance avec certaines personnes. Si mes terrains réguliers se situent en France et en Suisse, le réseau avec lequel j'interagis pour cette étude réunit des acteurs évoluant aux Pays-Bas, au Royaume-Uni, en Espagne, en Italie ou dans les pays scandinaves, qui circulent fréquemment d'un pays à l'autre pour leurs activités. Pour cette raison, je parle dans cet article de réseau européen du sabar.
} 


\section{Répertoire griotique, ethnique ou national : les évolutions locales du sabar}

Au Sénégal, le terme sabar désigne un instrument de musique, une danse et un moment de festivité dansée dont les caractéristiques musicologiques ou chorégraphiques ont été décrites par de nombreux anthropologues (Heath, 1994 ; Penna-Diaw, 2005 ; Tang, 2007 ; Dessertine, 2010 ; Neveu Kringelbach, 2013). Comme l'affirme le musicien cité en introduction, le sabar a longtemps été associé à la catégorie sociale des griots (géwël) et, encore aujourd'hui, les musiciens animant les fêtes de sabar sont en majorité des hommes, issus de familles s'identifiant comme griots. La danse était exécutée autrefois essentiellement par des femmes, à l'occasion de réunions d'associations féminines, de baptêmes, de mariages ou d'autres cérémonies survenant dans les cours des maisons (Penna-Diaw, 2005 ; Neveu Kringelbach, 2013 ; Seye, 2014). Le sabar et les cérémonies festives ${ }^{7}$ où il se performait représentaient des moments de représentation explosive du pouvoir sexuel féminin (Neveu-Kringelbach, 2013) et des espaces de transgression des normes de pudeur prévalant dans la société sénégalaise (Heath, 1994).

Sur un plan formel, cette danse énergique se compose de lancers de jambes et de sauts alternés, d'amples mouvements de bras, de "coupures " jouant sur des cassures saccadées du bassin et de soulèvement du pagne et vêtements du danseur (Penna-Diaw, 2005). Au-delà des caractéristiques gestuelles, les différents auteurs ayant analysé le sabar relatent que son essence réside surtout dans l'improvisation et la conversation qui relient le danseur au musicien, au public et au contexte de performance. Tout en puisant dans un répertoire de danses et dans une structure codifiée, cette tradition est ainsi sans cesse actualisée par le danseur dans ses improvisations et renouvelée par des appropriations de mouvements provenant $d^{\prime}$ autres pratiques locales (comme la lutte) ou des flux globaux.

Dans les dernières décennies, cette performance populaire a rencontré différentes transformations, l'amenant à sortir du registre des cérémonies privées, des savoir-faire griotiques et de l'ethnie wolof, pour être progressivement associée à une idée de patrimoine national. Une double " artification " (Heinich et Shapiro, 2012) du sabar est survenue à partir de l'indépendance, au travers d'échanges entre scènes locales et industries musicales globales. D'une part, une industrie musicale locale s'est créée autour de la rencontre entre les sonorités de sabar ou de tama $^{8}$ et les arrangements électroniques occidentaux, via la mise en place du genre mbalax à partir des années 1970. Incarné par des artistes comme Youssou N'Dour, Omar Pene, Thione Seck ou Viviane Chidid, ce genre est devenu extrêmement populaire au niveau local, mais il s'est également imposé sur les scènes de musique du monde, où il s'est érigé en genre musical sénégalais (Mangin, 2013). En parallèle de cette musique hybride, emblématique des fusions en cours dans les villes africaines depuis la colonisation, d'autres artistes comme Doudou

\footnotetext{
7 Les performances de sabar se tiennent fréquemment lors de fêtes appelées " sabar » lorsqu'elles sont diurnes, ou " tànnëbéers », lorsqu'elles sont nocturnes.

8 Le tama est un petit tambour d'aisselle qui est utilisé dans certaines danses du répertoire sabar et qui a été érigé dans les dernières décennies comme instrument national par les musiques populaires (Heath, 1994).
} 
N'Diaye Rose ont fait circuler une version plus épurée de la tradition percussive du sabar sur les scènes de musique du monde (Penna-Diaw, 2012). Ainsi, dans le genre hybride mbalax, ou dans sa version traditionaliste, le sabar est devenu le marqueur d'un son typiquement sénégalais.

D'autre part, outre la musique, la danse sabar s'est progressivement inscrite dans les représentations scéniques de la nation. Le nationalisme culturel s'est saisi de la danse pour affirmer des liens entre différentes régions du territoire au travers des ballets nationaux et de leurs chorégraphies folkloriques, entrainant une certaine fétichisation des différences ethniques (Castaldi, 2006 ; Kringelbach, 2012). Initialement basés sur des danses mandingues issues du Mali, de la Guinée ou de Casamance, ces ballets ont progressivement inclus le sabar dans leurs mises en scène (Tholon, 2009). Par la suite, les grands centres chorégraphiques afro-contemporains comme l'École des Sables l'ont aussi érigé en emblème d'une tradition locale à adapter scéniquement au moyen des techniques occidentales, et qu'ils transmettent et enseignent aussi à des stagiaires étrangers. Enfin, depuis le début des années 2000, des troupes de danse sont nées à Dakar, Saint Louis, Mbour, Kaolack ou Louga avec la volonté de promouvoir principalement les danses sabar, à l'instar de la compagnie African Ndiguel à Dakar, créée par un trio d'artistes masculins investis dans des carrières internationales.

De la sorte, la popularisation de la musique mbalax et des danses sabar a façonné une culture nationale chorégraphique commune à la jeunesse de Dakar et des villes du Nord-Est du Sénégal, en dialogue avec les politiques culturelles nationales. Au-delà de la caste des griots, de l'ethnie wolof, et des divisions de genre, les danseurs des troupes de sabar sont aujourd'hui des hommes et des femmes issus de plusieurs univers sociaux. Par leurs performances, ils déplacent le sabar dans différents espaces publics : les boîtes de nuit, les arènes de lutte (sport national et monde professionnel entretenant de nombreux échanges avec la danse), les vidéoclips ou les concerts d'artistes mbalax et les hauts-lieux de la création chorégraphique afro-contemporaine. De la sorte, comme dans d'autres États africains (Andrieu, 2014 ; Aterianus-Owanga, 2016), le développement des industries de la musique et de la danse a été corrélé avec la production d'une politique culturelle nationale et avec des circulations dans des marchés internationaux, conduisant à une artification de performances populaires.

Toutefois, la transformation progressive du sabar en répertoire national n'a pas été univoque et cette danse demeure aujourd'hui l'objet de visions ambivalentes. Le changement de contexte de réalisation du sabar, de la sphère privée au registre public, a conduit à ce qu'il devienne l'objet de nombreux " scandales " (Diop, 2012) car il visibilise et médiatise des transgressions aux normes locales de pudeur et de retenue. La pratique de la danse est ainsi fréquemment assimilée dans l'opinion publique à l'idée de prostitution (pour les femmes) ou à celle d'homosexualité (pour les hommes). La place majeure que le sabar occupe dans les débats publics et les procès pour obscénité qu'il rencontre (Briant, 2018) reflètent ainsi les tensions qui traversent la société sénégalaise contemporaine à propos des normes de respectabilité de genre (Diop, 2012).

Répertoire musical et dansé autrefois rattaché à des appartenances ethniques et griotiques, le sabar est ainsi progressivement devenu un emblème national ; 
simultanément, il a continué à faire l'objet de représentations ambigües, qui figurent les tensions traversant la nation sénégalaise. En sus de ces évolutions au niveau local, cette danse a aussi rencontré d'importantes modifications découlant de sa mise en circulation au sein du marché européen des danses africaines. Le façonnage de pédagogies propices à la transmission aux Européennes ${ }^{9}$ a renforcé certaines frontières distinguant le sabar sénégalais des répertoires mandingues et a conféré une dimension universelle à cette pratique, recomposant pour ce faire la tension entre tradition et individuation qui l'habite.

\section{Transmettre le sabar aux Européennes: des pédagogies entre " tradition " et individuation}

Depuis les années 1970, des cours de danses africaines réunissent des adeptes nombreux dans les villes européennes (Lefevre Mercier, 1987 ; Sawyer, 2006 ; Sieveking, 2002). Derrière l'intitulé générique de " danse africaine " (Lassibille, 2004), s'affirme en réalité tout un éventail de pratiques se revendiquant du registre de la " tradition " africaine, ou plus récemment des danses urbaines " modernes". Parmi ces pratiques, le sabar a intégré progressivement les studios de danse et les gymnases européens, d'abord sans être nommé spécifiquement. Durant les années 1980, il n'existait pas encore de classe spécifique de sabar et les premiers danseurs sénégalais qui s'installaient en Europe étaient amenés à enseigner des répertoires mandingues ou guinéens qui étaient alors plus populaires auprès de leurs élèves. Le sabar était perçu par les artistes de l'époque comme une pratique intransmissible aux toubabs (Occidentaux) ${ }^{10}$, car ancrée dans une logique d'improvisation et de "conversation " (waxtan) libre avec le musicien, ce que les pédagogies développées autour des danses mandingues ne permettaient pas - d'après mes interlocuteurs - d'inculquer à des néophytes. Hormis quelques mouvements de sabar glissés à l'intérieur des chorégraphies enseignées, les structures, rythmes et logiques chorégraphiques de la conversation dansée n'étaient pas explicités dans des enseignements consacrés.

Un changement survient à la fin des années 1990 et au début des années 2000. À l'époque, un nombre croissant de danseurs et de musiciens de sabar rejoignent I'Europe suite à des tournées artistiques ou des mariages avec des Européennes ; du fait de circuits de migration genrés, et comme la profession de musicien de sabar reste quasi exclusivement réservée aux hommes, ce sont depuis lors majoritairement des hommes qui enseignent le sabar en Europe, hormis quelques exceptions importantes que je cite plus bas. Ces artistes de sabar quittent alors un contexte dakarois où ce répertoire devient de plus en plus présent dans les vidéo-clips, les night-clubs et les scènes de musiques populaires, et où se développe l'enseignement du sabar. Dans le même temps, les adeptes européennes de danses africaines (majoritairement des femmes) deviennent plus enclines à explorer les spécificités culturelles et musicales dissi-

9 Les élèves de sabar d'Europe étant très majoritairement des femmes, j'opte pour féminiser les termes employés pour les désigner.

10 Souvent employée au Sénégal et dans d'autres pays ouest-africains comme synonyme de "Blancs " ou " Occidentaux ", la catégorie " toubab " revêt une dimension raciale, mais repose aussi sur des critères sociaux et culturels (voir Quashie, 2015). 
mulées derrière le label "danses africaines ". Autour de la rencontre entre ces artistes de sabar en migration et ces élèves passionnées, un marché spécifique se développe dans les villes européennes, au travers de stages et de cours de danse consacrés, de journées sénégalaises ou de festivités telles que celles se déroulant dans les rues de Dakar. Le sabar est alors présenté comme une danse proprement sénégalaise, à l'inverse d'autres répertoires mandingues que l'on retrouve dans différents pays.

Dans leurs enseignements, les artistes de sabar installés en Europe basent parfois leur légitimité artistique sur leur origine griotique (comme Maf Sing Sing évoqué plus haut), d'autres fois sur leur carrière au sein de grands ballets, ou plus simplement sur le fait qu'ils ont depuis leur enfance été bercés par les cérémonies de sabar, répertoire omniprésent dans les rues sénégalaises. Mais, au-delà de ces éléments, la profession de danseur et la légitimité à enseigner reposent aussi sur la question de la capacité à transmettre et de la pédagogie, centrale dans les carrières de ces artistes en migration. Dans un premier temps, une majorité des enseignants de sabar basaient leur pédagogie sur l'enseignement de chorégraphies, exécutées sur les rythmes les plus populaires du répertoire sabar, mais sans pour autant transmettre à leurs élèves des explications sur les rythmes, les logiques d'improvisation propres à chaque danse ou les contextes d'origine des fêtes de sabar. Progressivement, en interaction avec la demande de leurs élèves, quelques professeurs ont réformé leur enseignement afin de créer des pédagogies centrées sur la dimension improvisée de ces danses populaires et de rendre leurs codes rythmiques et kinesthésiques accessibles à des audiences non familières. Quoiqu'ils fassent figure d'exception, ils sont devenus extrêmement populaires en Europe et ont conféré une nouvelle portée à cette danse.

Le parcours deYama Wade est révélateur des arrangements qui ont été opérés dans cette démarche de transmission à des publics étrangers. Née et élevée à Dakar, dans une famille lébou du quartier populaire de la Médina - creuset des tannëbers et de cérémonies traditionnelles dont sont issues diverses danses du répertoire sabar - , elle apprend d'abord la danse dans le cadre familial, au travers de la transmission intergénérationnelle entre femmes. Parallèlement, elle intègre un célèbre ensemble chorégraphique de Dakar appelé " les ballets d'Afrique noire ", où elle apprend une série d'autres pratiques associées à I'aire mandingue, ainsi que les techniques et les mises en scène des ballets professionnels. Par ce canal, elle effectue des tournées en Europe, avant de s'installer à Paris au tournant des années 1990, où elle devient I'une des rares femmes à enseigner le sabar. À l'occasion d'un stage de danse, Yama Wade racontait ainsi à ses élèves la situation d'enseignement qu'elle découvrit lors de ses premiers voyages en Europe :

" Je suis arrivée en 92 ; ça fait longtemps. Il y avait deux profs de sabar, Y. et X. C'était juste ces deux profs. [...]X. m'avait dit: "je n'ai jamais transmis quoi que ce soit ; $j$ 'ai donné des petits trucs comme ça, mais les grands trucs, on ne donne pas". Donc quand je suis arrivée, j'ai trouvé ça : Y. qui donnait comme il pouvait, et X. qui donnait rien. Donc le travail a commencé là-bas [...]. " (Yama Wade, Lyon, avril 2017)

C'est lorsqu'elle est sollicitée pour remplacer un collègue professeur de sabar qu'elle prend conscience de l'impasse des méthodes développées 
jusqu'alors selon elle. Lors d'une interview, elle m'expliquait ainsi l'inconfort ressenti lorsqu'il s'est agi d'enseigner cette danse éminemment improvisée ${ }^{11}$ :

" Mais comment donner cette danse quoi ? Je savais déjà qu'il y avait pas d'esthétique, que c'était plutôt quelque chose que tu devais sortir de toi, que tu devais parler, tu devais discuter (waxtan). Mais comment je vais amener quelqu'un à discuter? Comment je dois faire pour que toi tu discutes avec le musicien ? [...] II [I'autre professeur] a rigolé,

il m'a dit: "tu es folle ! Ici c'est la France, c'est pas... tu vas pas le donner comme si tu dansais". II m'a dit: "tu ne danses pas, tu ne discutes pas, personne ne parle. On ne parle pas ; je t'ai pas demandé de faire parler l'autre. [...] Écoute-moi bien, je vais te dire comment tu dois faire : c'est les mouvements que tu donnes. Tu fais que des mouvements". " (Entretien avec Yama Wade, septembre 2018, Paris)

Se conformant d'abord à ces instructions d'enseignement,Yama découvre des élèves frustrées de ne pas saisir les structures qui leur permettraient de danser comme dans les festivités populaires. Elle décide alors de mettre en place une pédagogie alternative, qui lui vaut aujourd'hui le respect de nombreux élèves et pairs dans le milieu du sabar d'Europe. À l'inverse d'enseignements basés sur les chorégraphies, Yama Wade a façonné une pédagogie basée sur l'isolement de certains mouvements répétés en vue d'incorporer les justes positionnements et appuis du corps, sur l'enseignement d'une " grammaire " globale du sabar, et sur des " coachings " où elle s'entretient individuellement avec les élèves pour corriger leur technique et discuter de leurs ressentis. Reprenant fréquemment la métaphore linguistique, elle entend enseigner une "langue " à ses élèves, par l'explication de la " grammaire " d'ensemble du sabar et la transmission d'un " vocabulaire " de pas que les élèves pourront ensuite agencer selon leurs préférences. Son travail vise en d'autres termes à ce que les Européennes ne se contentent pas d'exécuter des suites de mouvements, mais soient en mesure d'entrer, de " parler " et de sortir du cercle de danse de façon autonome.

De la sorte, sa démarche positionne le sabar dans un entre-deux entre la recherche de développement individuel de ses élèves européennes et la puissance d'individuation qui serait au cœur des origines du sabar " traditionnel ". Yama présente aujourd'hui son enseignement comme une manière d'accéder à la "vérité à l'intérieur de soi ", et de se connecter à son "souffle intérieur ", en vue d'acquérir une libération individuelle. Elle explicitait ainsi cette conception à l'occasion d'un stage intensif :

"Vous êtes tous ici pour un but commun, c'est ça : acquérir la liberté, l'indépendance, développer quelque chose qui vient de toi, une vérité qui vient de toi, et c'est pour ça que vous êtes là. Et ça, on ne peut pas l'avoir si on vient comme prof et qu'on impose notre sabar. [...] C'est pas comme ça que ça marche chez nous. On nous a libérés, on nous a permis d'avoir chacun son sabar. C'est pour ça que chacun a son sabar. On nous a permis... Je pèse mes mots et j'insiste. II y a une démarche qui nous a permis de chercher notre sabar. Et c'est sur cette démarche qu'il faut aller, mais on ne peut pas imposer à quelqu'un son sabar. Dans le sabar il y a une partie où tu dois apprendre à développer ton sabar, à faire tes choix, à te libérer. " (Yama Wade, stage intensif de sabar, Lyon, avril 2017)

11 Ce témoignage comportait des termes en wolof, retranscrits ici en italique. 
En un sens, la méthode de Yama se présente comme intrinsèquement liée à la conception sénégalaise de la danse sabar, qui reposerait sur la création improvisée par le danseur à partir des codes de la danse et non sur la copie d'assemblages chorégraphiques existants. Parallèlement, elle reprend un lexique et des méthodologies rappelant fortement certaines démarches contemporaines de "développement personnel ", que l'on retrouve dans la pratique des spiritualités ou des disciplines corporelles autres en Europe, comme le yoga ou la méditation (Requilé, 2008). Face à certaines de ses élèves qui appréhendent le sabar comme un moyen de " se libérer ", Yama insiste sur la dimension curative de cette tradition. Elle revient aux origines de certaines danses dans des rites de possession comme le ndëp (Heath, 1994 ; Neveu Kringelbach, 2013), pour expliquer que "le sabar soigne " aussi bien physiquement que psychiquement. Sa technique de transmission du sabar se fabrique ainsi dans un équilibre entre la sauvegarde d'une tradition et d'un répertoire de danses originels, qu'il conviendrait de protéger des mutations contemporaines, et le don à ses élèves d'un moyen d'individuation au pouvoir universel.

Dans le sillage deYama Wade, de nombreux instructeurs affirment désormais des objectifs pédagogiques similaires : bien qu'ils emploient des vocabulaires et des outils différents, ils essaient de transmettre les logiques de l'interaction et de l'improvisation du sabar aux Occidentaux. C'est le cas de Sophie Sabar, une ancienne étudiante en ethnologie hollandaise, formée au sabar durant ses terrains à Dakar. Elle a mis en place à Amsterdam les premiers enseignements de danse mbalax et est devenue progressivement célèbre dans le réseau des danses africaines en Europe pour ses " conférences dansées ". Influencée par sa formation universitaire en ethnologie, elle a proposé ce format afin de répondre aux difficultés de certaines élèves à comprendre les modalités de l'improvisation. Elle y explicite le déroulement des fêtes de sabar, la succession des différents rythmes et les modalités d'entrée dans le cercle. D'autres professeurs comme Mbaye Sall, un danseur originaire de Louga et installé à Lausanne, consacrent une majeure partie de leur enseignement à accompagner la réalisation de solos improvisés par les élèves, plutôt qu'à l'acquisition de chorégraphies.

S'ils emploient des techniques variées, ces différents enseignants se rejoignent dans le souhait de transmettre aux Européennes des codes implicites pour évoluer dans le cercle et dialoguer avec le musicien. Ces diverses trajectoires et pédagogies ont fait du sabar un répertoire singulier à l'intérieur de l'offre des danses africaines, en mettant en exergue des logiques de conversation, d'individuation et d'improvisation propres à cette danse, mais que des acteurs de différentes origines seraient en mesure d'acquérir. Ils contribuent en ce sens à transformer le sabar en un langage corporel et musical " universel ".

Cependant, loin de se muer dans ce sillage en répertoire globalisé et déterritorialisé, le sabar demeure pour l'instant fortement rattaché à une idée de localité et d'identité sénégalaise. Comme nous allons le voir, ce maintien de la sénégalité du sabar survient au travers de diverses modalités d'inclusion ou d'exclusion de l'altérité. La première s'apparente à une entreprise de " sénégalisation " ou "d'africanisation " des adeptes européennes de sabar, passant par des circulations transgressant en apparence les frontières entre Sénégalais et étrangers. 


\section{Maintenir la sénégalité du sabar: circulations et conversions}

Même si les enseignants sénégalais de sabar estiment fréquemment que les Européennes peuvent difficilement développer une connaissance aussi fine du sabar que des Sénégalais nés dans une culture griotique, ils insistent souvent sur le fait que cette danse est un langage universel, légitimement accessible à toutes celles qui suivent une formation rigoureuse, et ils expriment leur souhait de partager ce bien culturel aux passionnées du Sénégal. Ils transmettent à leurs élèves un ensemble de savoirs chorégraphiques et culturels, les accompagnant dans des parcours qui s'apparentent sous certains aspects à des conversions vers une certaine "sénégalité ".

Cette sénégalisation des danseuses se manifeste d'abord, sur un plan des pratiques, par le port de tenues africaines en wax ou de tissus njaxas (étoffes juxtaposant différents tissus colorés) similaires à ceux portés par les baye fall' ${ }^{2}$, ainsi que par l'usage de certains termes et chansons en wolof. Ces pratiques qui s'observent dans les cours de danse se répandent parfois aussi en dehors de l'espace d'enseignement, et donnent lieu à de nouvelles esthétiques et soins du corps pour les élèves. L'investissement dans le sabar amène parfois certaines élèves à l'expression d'une passion plus profonde pour le Sénégal, qui les conduit à se consacrer exclusivement à ces répertoires sabar, alors décrits comme plus complexes que d'autres danses africaines. Cette " passion cognitive " (Roux et al., 2009) pour le Sénégal s'articule dans quelques cas avec des expériences intimes et des relations de couple avec des artistes musiciens ou danseurs sénégalais, que ces femmes vont accompagner dans leurs carrières et dans l'organisation d'activités de sabar en Europe ${ }^{13}$. Dans cet univers artistique éminemment genré, où des artistes africains majoritairement hommes interagissent avec des élèves européennes principalement femmes, le mariage avec un Sénégalais renforce en partie l'inclusion des femmes européennes au réseau social qui se met en place autour de l'enseignement du sabar en Europe. Ces différents éléments participent des parcours d'apprentissage des élèves de sabar, et alimentent les trajectoires de "conversion " à l'africanité (Raout et Chabloz, 2009) de certaines femmes européennes. Pour d'autres élèves, le sabar constitue un simple moment d'évasion et d'extraversion contrastant avec leur quotidien, mais sans pour autant se répercuter par des transformations sur le reste de leur existence.

Par ailleurs, au-delà d'acquisitions de connaissances, de transformations corporelles ou de nouvelles sociabilités, l'incorporation du sabar et l'appartenance à cet univers passent aussi par la réalisation de voyages fréquents au Sénégal. La connaissance de la terre d'origine du sabar apparait dans les discours des élèves et des enseignants comme un prérequis incontournable. Fatou Woré, une célèbre instructrice de sabar, témoignait de cette importance de partir et de " durer " au Sénégal pour approcher les finesses de la danse, alors

12 Au Sénégal, le bayefallisme désigne une branche dérivée du mouridisme. Autrefois marginale (Pezeril, 2008), elle éveille aujourd'hui l'engouement des jeunes hommes et artistes de Dakar (Havard, 2001).

13 Cet univers artistique en migration comporte des similitudes avec celui des danseurs africains contemporains (Despres, 2015). 
que je l'interrogeais sur le fait que des toubabs puissent danser le sabar comme les Sénégalais:

"Bien sûr ! J'ai une élève qui habite à Nagoya, au Japon, Maoshi. Maintenant, on l'appelle Fatou Woré du Japon ; parce qu'elle danse super bien. Tu peux le faire, mais il faut que tu prennes du temps au Sénégal. » (Entretien avec Fatou Woré, mai 2017, Lyon)

Comme l'expliquent les professeurs de sabar dans leurs enseignements, l'apprentissage du sabar par les Occidentaux requiert de connaître le contexte d'origine de cette danse, d'expérimenter le fait de danser sur les plages sénégalaises et de fréquenter les fêtes de Dakar. Autour de cette importance du voyage en Afrique, des danseurs ou musiciens organisent plusieurs fois par an des stages de sabar à Dakar ou dans d'autres villes et sites touristiques sénégalais (Louga, Mbour, Saint Louis, Saly). Ces stages d'une à deux semaines fournissent aux participantes une formation de danse intensive (plusieurs heures par jour), et organisent leur logement, restauration et excursions touristiques, y compris dans des troupes de danse ou des fêtes de sabar. Durant ces voyages, les danseurs et les musiciens sénégalais se muent en guides touristiques et en intermédiaires de la rencontre des Européennes avec la " culture " sénégalaise (Aterianus-Owanga, à paraître).

Ces circulations corollaires à l'enseignement du sabar participent à maintenir la localisation du sabar dans son contexte territorial sénégalais. Parallèlement, elles répondent aussi à une représentation, généralement partagée par les danseurs de sabar, selon laquelle l'entretien de leurs compétences et connaissances chorégraphiques serait dépendant de retours fréquents au Sénégal. En plus d'être considéré comme le site d'origine du sabar, le Sénégal demeure pour les acteurs de ce champ le lieu unique d'innovation de ce répertoire chorégraphique et musical (Aterianus-Owanga, 2018). En Europe, il n'existe pas de troupes de sabar répétant de façon régulière, et la faible possibilité de professionnalisation atténue l'effet d'émulation et de compétition au travers duquel se renouvelle le répertoire. Pour ces artistes, les retours récurrents au Sénégal sont donc vus comme la source des savoirs et des innovations qui nourriront leurs enseignements en Europe. Dans ce sillage, le sabar reste ainsi rattaché à un territoire délimité, tout en circulant au-delà de ses attaches originelles et auprès de nouveaux publics, en d'autres termes, en se transnationalisant.

Via ces circulations et ces conversions, les artistes de sabar dansent et font danser les Européennes sur les frontières apparemment flexibles, poreuses et inclusives de l'identité sénégalaise et du réseau du sabar. Néanmoins, en deçà de ces échanges "transculturels ", I'observation des polémiques qui traversent la scène sabar soulignent que des logiques d'exclusion et de marquage de différenciation persistent en parallèle. L'observation des enjeux de rétention du savoir dansé révèle la manière dont le maintien de certaines frontières s'articule à des rapports de pouvoir et des inégalités enracinés dans I'histoire et la situation postcoloniale. 


\section{Au-delà de l'appropriation : frontières et rapports de pouvoir}

En février 2017, la diffusion sur Facebook d'une vidéo intitulée "Cultures Vultures vs the real thing "générait une discussion en ligne parmi quelques adeptes de danses africaines et de sabar en France. Partagée initialement par un abonné dénommé "The African-American community ", puis reprise par différents abonnés Facebook, la publication consistait en un montage de deux vidéos : la première, décrite comme l'expression d'une "culture originale ", donnait à voir la performance d'un groupe de femmes africaines, vêtues de pagnes et de T-shirts, exécutant des mouvements synchronisés dans une rue en terre battue, devant un petit rassemblement de spectateurs. Sur la seconde vidéo, un groupe de femmes blanches vêtues de tenues en wax effectuait dans la rue d'une ville européenne une performance de danses sabar, accompagnées en arrière-plan par un groupe de musiciens noirs, devant un public occidental qui prenait des photos ou observait la performance.

Sur les deux vidéos, ni le lieu, ni les danseuses, ni les spectateurs concernés n'étaient caractérisés par un marqueur descriptif lié à une origine nationale, ethnique, ou spatiale. Même si aucun des commentateurs du montage n'identifiait la provenance de la première vidéo - celle capturée en Afrique -, tous s'accordaient pour dire qu'il ne s'agissait pas d'une danse sénégalaise, et qu'elle était issue d'une autre région du continent. La vidéo des danseuses occidentales n'était pas non plus identifiée, mais la connaissance de certaines personnes y figurant me permet de préciser qu'il s'agit d'une vidéo prise en Allemagne, d'un groupe d'élèves qui exécutent une danse du répertoire sabar nommée yaaba.

L'effet principal du montage était d'opposer des danseuses dites " originales " ou " authentiques", en l'occurrence des danseuses africaines, à des danseuses dites " vautoures " ou voleuses de culture, les femmes blanches s'adonnant à la pratique de danses africaines. Une grande partie des commentaires du monde anglophone louait la richesse et l'âme des danses africaines "originales ", et raillait la performance des danseuses occidentales. À l'inverse, quelques commentaires désapprouvaient la critique de ces femmes qui « embrassent la culture des autres " et relevaient leurs efforts pour accomplir leur performance.

Au-delà du monde anglophone, je découvris cette vidéo via le fil de discussion d'adeptes françaises de " danses africaines " qui discutèrent la publication : plusieurs regrettaient que le débat se polarise autour d'une opposition raciale et appelaient à ne pas confondre échange culturel et pillage culturel. Une professeure de danses sénégalaises réagissait pour sa part en décrivant le "ridicule " de la performance des danseuses blanches. Mais plutôt que de blâmer les danseuses elles-mêmes, elle critiquait la piètre qualité de l'enseignement qu'elles avaient dû recevoir, et qui les amenait ainsi à " gesticuler ".

Comme le montre cet exemple, la transmission du sabar auprès du public occidental soulève des polémiques qui se rattachent en partie à ce qui est appréhendé depuis quelques années en termes d'appropriation culturelle. À partir des années 1980, différents secteurs des industries culturelles ont débattu autour de cette idée, employée pour désigner un phénomène d'adoption de traits culturels d'un groupe dominé par les acteurs d'un groupe dominant. Née dans 
le contexte nord-américain dans les années 1990, elle est devenue le support de théories critiques et militantes (Hook, 1992 ; Root, 1995), qui s'attachent à mettre en exergue les rapports de domination, d'exotisation de l'altérité et d'exploitation sous-jacents à certains types d'emprunts culturels. Récemment popularisée dans les médias français autour d'affaires liées au milieu musical ${ }^{14}$, la notion d'appropriation culturelle a pour mérite de mettre en évidence dans le débat public les inégalités existant dans le contrôle des ressources culturelles et les héritages de schémas coloniaux et exotisants qui sous-tendent les industries culturelles.

Toutefois, ce concept s'avère réducteur s'il s'agit d'appréhender les dynamiques d'emprunt culturel et les rapports de pouvoir auxquels elles s'associent. D'une part, en envisageant les "cultures " comme des blocs homogènes, il occulte les dimensions fondamentalement hybride, plastique et hétérogène des pratiques culturelles, ainsi que les emprunts qui sont au cœur de tous les processus de production culturelle, particulièrement dans le domaine des musiques (Martin, 2014). En outre, son application s'accompagne de lectures réductrices des rapports de pouvoir charriés par la circulation de biens culturels: elles occultent le rôle des stratégies des différents acteurs impliqués dans ces processus et les interactions dans lesquelles ils sont pris, en réduisant les échanges en présence à un affrontement entre des blocs essentialisés (Noirs/ Blancs, Nord/Sud, dominants/dominés).

Ainsi dans cet exemple, la pratique des danses africaines par les Occidentales est présentée par certains commentateurs de la publication Facebook comme un " pillage " des cultures, mettant en opposition binaire et antagonique des " cultures " africaine et occidentale, et polarisant les enjeux du débat autour de l'argument racial. À l'inverse de cette lecture, l'examen ethnographique à l'échelle des réseaux d'individus révèle les imbrications de motifs plus complexes qui amènent à ces tensions autour de la mise en circulation du sabar.

Loin d'être récentes, les résistances à la diffusion du sabar auprès des Occidentaux surgissent dès les premières tentatives de création de pédagogies alternatives, comme en témoignent les critiques rencontrées par Yama Wade. Cette dernière relate fréquemment les violentes réprimandes qu'elle reçut de ses pairs danseurs et musiciens en Europe lorsqu'elle commença à développer sa pédagogie. Ceux-ci lui reprochaient de donner aux toubabs les " secrets du sabar " et de divulguer des savoirs censés rester entre les mains des Sénégalais. Yama raconte également comment certains de ses prédécesseurs refusaient alors d'enseigner aux Européennes certains pas de base du sabar, sous prétexte que leur compréhension requérait un certain niveau dans la pratique, ou que les toubabs ne seraient pas en mesure de les acquérir.

Des discours et réticences similaires se retrouvent aujourd'hui chez une partie des enseignants de sabar, en raison d'explications diverses. La première se trouve dans la persistance de préjugés raciaux relatifs aux compétences chorégraphiques et à la maîtrise de cette danse. Ces préjugés sont parfois

14 On pensera notamment au cas du twerk de Miley Cirus (Djavadzadeh, 2016) ou aux mises en scène de la chanteuse Madonna avec une tenue berbère lors des MTV Music Awards de 2018. 
rendus manifestes dans les stages, par des discussions (en aparté et en wolof) entre musiciens à propos des maladresses des élèves, et par une tendance assez générale à distinguer des manières de se comporter ou de danser qui seraient "toubabs " ou d'autres qui seraient "sénégalaises ". Cette pensée formulée au travers de terminologies raciales considère qu'au-delà d'un niveau superficiel, les Occidentaux sont incapables de développer une compréhension du " sabar pur ", un style propre ${ }^{15}$ et une communication libre avec le musicien, ces éléments caractéristiques du bon danseur que seuls les Sénégalais posséderaient (et en réalité, seuls quelques Sénégalais). Le manque de style, les lacunes ou les maladresses corporelles des élèves occidentales sont ainsi appréhendés par quelques enseignants comme la résultante de frontières et de différences entre "toubabs " et Sénégalais, où s'entrecroisent indistinctement les aspects raciaux et culturels. Quelles que soient les années d'enseignement, ces différences voueraient à l'échec le développement d'un enseignement plus approfondi, et rendraient impossible une compréhension intégrale de cette tradition. Ces idées de compétences au sabar inégalement réparties en fonction des appartenances raciales ou culturelles ne ciblent pas seulement les Occidentaux et concernent également les Africains non sénégalais : ces derniers sont considérés comme plus outillés pour apprendre cette danse africaine, et en quelque sorte supérieurs aux toubabs dans l'échelle implicite des aptitudes à danser le sabar, car plus proches culturellement des Sénégalais. Mais ils demeureraient cependant toujours moins doués que les Sénégalais, car extérieurs au vivier culturel dans lequel s'enracinerait le sabar. Racisme, culturalisme et nationalisme s'entremêlent ainsi dans les représentations relatives à l'enseignement du sabar en Europe.

La seconde explication à ces critiques et réticences à transmettre les arcanes du sabar aux étrangers réside dans la dimension économique, et dans la question des bénéficiaires des circulations monétaires induites par la diffusion du sabar en dehors du Sénégal. En effet, la plupart des enseignants sénégalais de sabar acceptent que des Européens ou des Africains non sénégalais apprennent le sabar, et ils expriment de la joie et de la fierté à les voir acquérir un bon niveau dans cette danse. En réalité, s'ils sont réticents à ce que les nonSénégalais en maîtrisent les complexités, c'est car ils ne veulent pas qu'ils en viennent à I'enseigner eux-mêmes ${ }^{16}$. Une célèbre danseuse de Dakar m'exposait ainsi ce point de vue, réagissant plus précisément au fait que quelques toubabs viennent apprendre le sabar au Sénégal pour l'exporter ensuite vers les studios de danse européens, dans des cours payants :

"Normalement, c'est nous, c'est les danseurs sénégalais, qui apportent la danse en Europe. Donc si on accepte que les toubabs viennent ici, apprendre notre danse, d'aller en France ou en Amérique et de gagner ce que nous on doit gagner, ça, ça me touche un peu. Ça me fait mal. Je dis la vérité. [...] Oui, est-ce que vous les toubabs, vous acceptez

15 Une enseignante sénégalaise de sabar m'expliquait ainsi : "Moi je vois vraiment des Européennes qui dansent très bien, mais il y a quelque chose qui manque, c'est avec nous, je sais pas pourquoi, c'est avec nous [les Sénégalais]. [...]Tu dois avoir ton propre style. Quelque chose qui est en toi, qui t'appartient, que quelqu'un d'autre n'a pas. C'est ça que les Occidentaux n'ont pas encore. " (H., Lyon, mars 2017)

$16 \mathrm{Si}$ des rapports de concurrence existent entre danseurs de sabar en Europe, la question du " vol " des pas ne semble pas centrale dans les processus de création, à I'inverse du contexte malien (Djebbari, 2014). 
que votre culture, nous on vient la prendre là-bas, en Suisse ou je sais pas, de l'amener ici, de donner aux Sénégalais, les Sénégalais gagnent de l'argent, et vous vous gagnez rien du tout? Est-ce que vous acceptez ça ? [...] Les Européens gagnent ce que nous on doit gagner. Ça, je l'accepte même pas. Vraiment ça me fait mal, et je le dis haut et fort. "

(N., Dakar, février 2018)

Dans cet exemple, la revendication de propriété culturelle sur le sabar (" notre culture ") est clairement corrélée avec la question des entrées financières induites par la mise en circulation de ces biens. Pour cette raison, le point le plus épineux de ces polémiques porte sur la question des Européennes qui enseignent elles-mêmes le sabar, et accèdent aux rentrées financières (même minimes) induites par sa transmission en Europe. Derrière cet argument économique, c'est globalement la question de l'inégalité d'accès aux moyens de communication et à la connaissance des réseaux culturels en Europe qui est dénoncée : il est reproché aux Occidentaux leur commercialisation d'un bien culturel appartenant à des individus évoluant dans un contexte de précarité, souffrant d'une absence de soutien institutionnel au Sénégal et d'une expérience de marginalisation dans le contexte européen.

Parallèlement, les discours de certains danseurs ou musiciens sénégalais à propos des limites à imposer dans la transmission des savoirs résonnent de façon implicite avec la critique des relations postcoloniales et la perpétuation des logiques d'exploitation entre la France et le Sénégal. J'ai pu observer ces motifs dans les discussions entre musiciens et danseurs sur l'enseignement, mais aussi dans certaines réactions engendrées par ma propre démarche de chercheure sur cet objet. En effet, plusieurs interlocuteurs regrettaient que ce soit à nouveau une toubab qui se charge d'effectuer une recherche sur une " tradition " qui devrait être décrite par ses acteurs et détenteurs eux-mêmes, et que les financements de telles recherches ne soient pas plutôt octroyés à des Sénégalais. D'autres sous-entendaient que la réalisation d'entretiens ou d'observations ethnographiques devait être accompagnée d'une rétribution financière. Ma première rencontre avec un danseur installé en Europe apporte des éléments révélateurs à propos de l'intrication des dimensions économiques et des enjeux postcoloniaux dans ce contexte, comme en témoignent ces notes de terrain :

Dès le début de notre discussion, après que je lui ai explicité ma démarche de recherche, X. émet des réticences à l'idée d'un format entretien, et il refuse que je lui pose des questions sur son parcours ou que je l'enregistre. II me dit qu'il veut bien " partager " des choses avec moi et "échanger ", mais que pour me parler de lui, il faudrait qu'il me connaisse depuis longtemps ou alors que je le paye. Nous parvenons finalement à converser, après que j'ai abandonné l'idée de justifier ma recherche et de poser des questions, pour me contenter de le laisser partager ce qu'il souhaite, en le relançant à certains moments, tout en le laissant m'interroger également ou me donner des conseils. [...] Au début de la conversation, il me dit qu'il pense que le sabar est pour tout le monde, qu'« il n'a pas de couleur, blanc, noir, car tout le monde a le même sang ".

Au terme d'une longue discussion sur nos parcours respectifs, il en vient à me confier que lorsqu'il transmet ses connaissances à des 0ccidentaux, il est heureux et fier, mais qu'il a besoin de " sentir l'argent de la personne, bien froid dans [sa] poche, et dans [son] ventre ". Parce qu'" on a pardonné le passé, mais on n'oublie pas ".

(Notes de terrain, mai 2017) 
Au-delà des enjeux de réflexivité, cette situation de résistance à l'entretien souligne l'imbrication de dimensions politiques, économiques et culturelles dans la transmission de cette danse, et la manière dont la maîtrise des flux économiques est conçue par ces artistes comme un moyen de se prémunir de rapports d'exploitation pensés en termes de binarité Nord/Sud, ou Noirs/ Blancs, et rattachés à une histoire marquée par la colonisation et l'esclavage. En d'autres termes, la transaction économique, posée comme la condition de la transmission des biens et savoirs culturels, répond à des rapports de pouvoir entre Europe et Afrique que ces individus veulent inverser. Des relations de longue durée conduisent dans certains cas à atténuer ces attentes économiques et à contrebalancer ces inégalités, par la mise en place de rapports de confiance, et d'activités communes.

Alors que les frontières culturelles entre le Sénégal et l'Europe semblent flexibles et inclusives durant certains moments, cet exemple montre qu'elles deviennent rigides et exclusives sous d'autres aspects, notamment en raison de persistantes distinctions raciales et d'inégalités économiques. Cet imbroglio de relations et de transactions qui sous-tend les situations d'enseignement prend sa source dans la dénonciation d'un rapport d'exploitation ou d'inégalités Noir/ Blanc, mais il est aussi complexifié par l'existence de relations interpersonnelles, particulièrement dans le registre intime. En effet, et comme indiqué plus haut, le monde des danses africaines est en partie soutenu par l'existence de couples mixtes qui facilitent la migration, l'acquisition de visas longue durée, la mise en place d'enseignements et d'activités pour les danseurs. Si ces relations conjugales entre artistes sénégalais et femmes européennes sont parfois décrites comme un véritable partenariat, elles sont aussi d'autres fois appréhendées comme le résultat d'intérêts mutuels, et notamment des stratégies de captation de savoirs par les élèves. Quelques artistes hommes interrogés racontent comment leur statut d'artiste musicien ou danseur africain les rend attractifs aux yeux des femmes occidentales, motivées selon eux par le désir d'acquérir une proximité particulière avec la culture sénégalaise et les savoirs dont ils seraient détenteurs :

"[En Europe], il y a des femmes qui aiment beaucoup la culture africaine, elles veulent beaucoup le faire à la maison avec quelqu'un qu'elle aime. Tu vois? Elles aiment beaucoup être avec le danseur 24h/24.

AA : Tu as l'impression que se mettre en couple, c'est une manière d'acquérir la culture sénégalaise aussi?

K. : Oui, d'un côté. Donc il y en a beaucoup qui se sont épousées avec des danseurs, mais après ça marche plus. Parce qu'elle dit: "Oui, on s'est épousés, parce que je voudrais qu'on s'échange beaucoup à la maison. Mais tu n'as pas mon temps, tu es sur le lit, ou bien au salon, ou bien sur Facebook. Tu n'as pas le temps de me montrer les choses". Et vraiment à partir de cela, les problèmes commencent à venir petit à petit, tu vois ?"

(K., danseur, Lausanne, octobre 2018)

Au-delà du témoignage de ce danseur, les discussions informelles avec des élèves de danses africaines ou avec des artistes suggèrent que le fait d'être en couple avec un artiste de renom serait employé par certaines femmes occidentales comme moyen de se rapprocher et de s'approprier un univers culturel convoité. La relation entre quelques artistes hommes sénégalais et leurs élèves occidentales met ainsi en jeu des circulations de biens économiques, de capital 
culturel et de notoriété. Pour ces raisons, bien que les hommes sénégalais engagés dans des relations avec des femmes occidentales soient fréquemment critiqués pour la nature "intéressée " de leurs comportements, la plupart des acteurs de ce champ reconnaissent que cet intérêt est bilatéral, et que loin d'être victimes de ces relations, les femmes européennes en tireraient également un parti avantageux.

Cet entrelacement de différents rapports sociaux de pouvoir (de genre, de classe, de race) souligne deux points importants pour notre réflexion. Sur un premier plan, la dénonciation de "l'appropriation culturelle " du sabar ne se corrèle pas aux mêmes enjeux et débats que dans le contexte des musiques afroaméricaines. Dans les musiques afro-américaines, les polémiques survenues autour de la reprise d'esthétiques noires par des pop stars blanches comme Miley Cirus se développent sur fond de tensions relatives au "mythe d'une Amérique post-raciale où la race serait sans importance " et contestent le profit commercial d'artistes qui nient l'expérience des groupes minoritaires (Djavadzadeh, 2016). Dans le monde du sabar, si ces controverses semblent en apparence se nicher dans des systèmes d'inégalité et des antagonismes raciaux similaires, il apparait qu'elles reposent sur les logiques de relations et de transactions propres à ce réseau d'individus : elles trouvent leurs sources dans des inégalités économiques, des tensions liées à l'histoire coloniale et postcoloniale, et des rapports de pouvoir à l'échelle des individus où s'entrelacent des dimensions de genre et de classe. Dans ce contexte, en deçà (ou au-delà ?) de l'appropriation culturelle, c'est bien les transactions et les relations intersectionnelles en présence qui doivent être prises en compte, à l'échelle interindividuelle et donc sur un plan ethnographique, pour comprendre les résistances à l'appropriation culturelle et les affirmations de frontières, d'identités ou de propriétés qui les accompagnent.

Sur un second plan, l'examen de ces controverses relatives à l'appropriation du sabar par les Occidentaux confirme l'existence d'une connexion indissociable entre frontières et pouvoir. Comme le notent les théoriciens des frontières entre groupes sociaux, ces dernières " jouent un rôle important dans la création des inégalités et dans l'exercice du pouvoir " (Lamont et. al., 2001 : 15341). Le cas du monde du sabar atteste que l'affirmation de frontières entre des groupes sociaux (les Sénégalais et les " autres ", Africains ou Occidentaux) et de droits inégaux à profiter des ressources culturelles qui leur appartiendraient peut être utilisée afin de contester des inégalités en place ou des rapports de domination : en transmettant le sabar aux Européens et en affirmant la propriété des Sénégalais sur ce répertoire culturel, les danseurs et danseuses sénégalais utilisent la frontière (entre Sénégalais et non-Sénégalais, entre Européens et Africains) pour rendre visibles les rapports de domination hérités de I'histoire coloniale et pour agir à leur échelle sur la structuration inégalitaire des relations entre l'Afrique et I'Europe. Finalement, I'approche ethnographique de cet univers artistique en migration souligne la dimension relationnelle et situationnelle de la frontière : dissoute dans certaines situations, elle est réaffirmée dans d'autres, lorsque des rapports de pouvoir sont mis en jeu. 


\section{Conclusion}

Performance féminine associée aux groupes ethniques wolof et lébou du Sénégal et à la caste des griots, le sabar a rencontré différentes transformations dans les dernières décennies qui ont reconfiguré les logiques de frontières et d'appartenance charriées par cette performance. Cet article s'est attaché à retracer ce processus au niveau local et transnational. Dans un premier temps, le sabar a été institué comme un bien culturel sénégalais, au travers des politiques culturelles nationales et des circulations transnationales des danseurs et des musiciens. Par la suite, cette performance est devenue le support d'identification d'une partie de la jeunesse urbaine et des troupes de danse populaires de Dakar. Les trajectoires de migration de ces danseurs ont conduit à déterritorialiser la pratique du sabar vers d'autres espaces et d'autres publics. Étroitement associé à l'idée d'identité et de valeurs sénégalaises, ce répertoire dansé est désormais transmis à un public d'Occidentaux, essentiellement des femmes, à l'intérieur du marché des danses africaines. La transmission du sabar au-delà des frontières sénégalaises amène alors à des jeux d'inclusion et d'exclusion de l'altérité dans les frontières de la nation sénégalaise.

J'ai proposé ici une approche des techniques de transmission du sabar, des interactions et des tensions qu'elles génèrent en vue d'interroger la production relationnelle et situationnelle des frontières en contexte transnational. Nous avons ainsi vu dans un premier temps que la transmission du sabar en Europe a conduit à la mise en place de pédagogies hybrides et " universelles " permettant de négocier les différences culturelles au travers d'arrangements entre des influences diverses. Mais contrairement à une idée de déterritorialisation consécutive à cette création de pédagogies " universelles ", l'enseignement du sabar en migration recompose aussi les identifications et les frontières nationales, par des jeux d'inclusion des élèves qui s'intéressent à cette performance. Finalement, les polémiques générées de façon indirecte autour de la transmission du sabar aux non-Sénégalais révèlent que l'existence de situations d'inclusion ne dissout pas le terreau d'inégalités sur lequel s'est construit ce monde artistique, et qui conduit à la réaffirmation de frontières entre les artistes sénégalais et leurs élèves.

"Sabar, ma tradition " : la citation présentée en amorce de mon article reformulait cette idée commune qui suppose l'interdépendance entre une tradition dansée, une culture et un groupe social qui en serait le dépositaire. Dans la continuité de Fredrik Barth, I'examen du monde du sabar en migration a démontré que la persistance de ces trois éléments (danse, culture, groupe social) repose tout autant sur l'utilisation de différences culturelles existantes que sur l'expérience de frontières et de relations avec d'autres groupes sociaux, notamment en situation de migration. En fonction des rencontres, des contextes et des enjeux de pouvoir, le sabar se présente alors tantôt comme un marqueur de frontières culturelles, tantôt comme un moyen de tisser un pont transcendant ces frontières. 


\section{Références bibliographiques}

Amselle Jean-Loup et Elikia M'Bokolo (Éds.) (1985) Au cœur de l'ethnie : ethnies, tribalisme et État en Afrique, Paris, La Découverte, 227 p.

Anderson Benedict (1996) L'imaginaire national. Réflexions sur l'origine et l'essor du nationalisme, Paris, La Découverte, $224 \mathrm{p}$.

Andrieu Sarah (2014) Les valeurs de la création chorégraphique ouest africaine, Volume !, 10 (1), pp. 89-111.

Apprill Christophe (2017) Patrimonialisation de la danse tango : une "tradition" au prisme de sa déterritorialisation, Autrepart, 78-79, pp. 145-162.

Askew Kelly (2006) Performing the Nation. Swahili Music and Cultural Politics in Tanzania, Chicago, University of Chicago Press, 392 p.

Aterianus-Owanga Alice (à paraître) "Kaay fecc!" (Come dance!) Economic, cultural and intimate flows in the "dance tourism" of sabar (Senegal-Europe), in Mayke Kaag, Guive Khan-Mohammad and Stefan Schmid Eds., Destination Africa. Contemporary Africa as a Global Meeting Point, Leiden, Brill.

Aterianus-Owanga Alice (2018) Le tànnëbéer multisitué. Danses et communauté émotionnelle des fêtes sénégalaises en migration, Socio-anthropologie, 38, pp. 89-108.

Aterianus-Owanga Alice (2016) Un Janus à deux visages. Patrimonialisations du religieux initiatique et discours de la tradition dans les musiques " tradimodernes " du Gabon, Autrepart, 78-79 (2-3), pp. 103-124.

Averill Gage (1994) "Mezanmi, Kouman Nou Ye? My Friends, How Are You?": Musical Constructions of the Haitian Transnation, Diaspora: A Journal of Transnational Studies, 3 (3), pp. 253-271.

Barth Fredrik (1995) Les groupes ethniques et leurs frontières, in Philippe Poutignat et Jocelyne Streiff-Fenard Éds., Théories de l'ethnicité, Paris, Presses Universitaires de France, pp. 203-249.

Bizas Eleni (2014) Learning Senegalese Sabar: Dancers and Embodiment in New York and Dakar, London, Berghahn Books, 168 p.

BohIman Philip V. (2011) When Migration Ends, When Music Ceases, Music and Arts in Action, 3 (3), pp. 148-166.

Briant Sarah (2018) Mettre en scène l'obscène. Les usages chorégraphiques du sabar par la scène contemporaine dakaroise, in Federica Fratagnoli et Mahalia Lassibille Éds., Danser contemporain : Gestes croisés d'Afrique et d'Asie du sud, Montpellier, Deuxième époque, pp. 137-168.

Castaldi Francesca (2006) Choreographies of African Identities: Négritude, Dance, and the National Ballet of Senegal, Urbana and Chicago, University of Illinois Press, $246 \mathrm{p}$.

Despres Altaïr (2015) Et la femme créa I'homme. Les transactions culturelles intimes dans la danse contemporaine africaine, Sociologie, 6 (3), pp. 263-278.

Dessertine Audrey (2010) Une initiation diffuse à la sexualité, Civilisations. Revue internationale d'anthropologie et de sciences humaines, 59 (1), pp. 89-108.

Diop Awa (2012) Identités féminines " transgressives " au Sénégal : un rapport ambivalent à la glocalisation, Thèse de doctorat, Université de Bordeaux 2. 
Djavadzadeh Keivan (2016) Les masques noirs des pop stars blanches. Miley Cyrus et les politiques de l'appropriation culturelle, Raisons politiques, 62, pp. 21-33.

Djebbari Élina (2014) Voler, donner, transmettre. Propriété et appropriation chez les artistes de ballet du Mali, Volume !, 102, pp. 173-193.

Havard Jean-François (2001) Ethos " bul faale " et nouvelles figures de la réussite au Sénégal, Politique africaine, 82 (2), pp. 63-77.

Heath Deborah (1994) The politics of appropriateness and appropriation: recontextualizing women's dance in urban Senegal, American ethnologist, 21, pp. 88-103.

Heinich Nathalie et Shapiro Roberta (Dir.) (2012) De I'artification : enquêtes sur le passage à l'art, Paris, EHESS, $336 \mathrm{p}$.

Hooks bell (1992) Black looks: Race and representation, Bosta, MM, South East Press, $200 \mathrm{p}$.

Hughes-Freeland Felicia (2008) Embodied communities: dance traditions and change in Java, New York, Berghahn Books, 286 p.

Jeanpierre Laurent (2010) Frontière, in Christin Olivier Éd. Dictionnaire des concepts nomades en sciences humaines, Paris, Métailié, pp. 157-169.

Lamont Michèle and Molnár Virág (2002) The study of boundaries in the social sciences, Annual review of sociology, 28 (1), pp. 167-195.

Lamont Michèle, Pendergrass Sabrina and Pachucki Mark C. (2001) Symbolic boundaries, International encyclopedia of the social and behavioral sciences, 23, pp. 15341-15347.

Lassibille Mahalia (2004) "La danse africaine ", une catégorie à déconstruire, Cahiers d'études africaines, 175 (3), pp. 681-690.

Lefevre Mercier Isabelle (1987) Les danses noires de l'Occident : analyse sociologique et interprétation de l'apparition en Europe d'une activité corporelle dansée : I'exemple des cours africains de Paris, Thèse de doctorat, Université Paris Diderot - Paris 7.

Mangin Timothy (2013) Mbalax: Cosmopolitanism in Senegalese Urban Popular Music, PhD Dissertation, University of Columbia.

Martin Denis-Constant (2014) Attention, une musique peut en cacher une autre. L'appropriation, $A$ et $\Omega$ de la création, Volume !, 10, pp. 47-67.

Mitchell J. Clyde (1983) Case and situation analysis 1, The sociological review, 31 (2), pp. 187-211.

Neveu Kringelbach Hélène (2013) Dance Circles: Movement, Morality and SelfFashioning in Urban Senegal, London, London, Berghahn Books, 252 p.

Neveu Kringelbach Hélène (2012) Moving shadows of Casamance: performance and regionalism in Senegal, in Hélène Neveu-Kringelbach and Jonathan Skinner, Dancing Cultures: Globalization, Tourism and Identity in the Anthropology of Dance, New York, Berghahn Books, pp. 143-160.

Penna-Diaw Luciana (2012) Doudou Ndiaye Rose, I'artiste caméléon, Cahiers d'ethnomusicologie. Anciennement Cahiers de musiques traditionnelles, 25, pp. 41-54. 
Penna-Diaw Luciana (2005) La danse sabar, une expression de l'identité féminine chez les Wolof du Sénégal, Cahiers d'ethnomusicologie. Anciennement Cahiers de musiques traditionnelles, 18, pp. 201-215.

Pezeril Charlotte (2008) Islam, mysticisme et marginalité : les Baay Faal du Sénégal, Paris, L'Harmattan, 320 p.

Quashie Hélène (2015) La " blanchité " au miroir de l'africanité, Cahiers d'études africaines, 220 (4), pp. 761-786.

Raout Julien et Chabloz Nadège (2009) Corps et âmes. Conversions touristiques à l'africanité, Cahiers d'études africaines, 193-194, pp. 7-26.

Requilé Élise (2008) Entre souci de soi et réenchantement subjectif. Sens et portée du développement personnel, Mouvements, 54, pp. 65-77.

Root Deborah (1995) Cannibal Culture:Art, Appropriation and the Commodification of Culture, Boulder, Westview Press, 239 p.

Roux Jacques, Charvolin Florian et Dumain Aurélie (2009) Les " passions cognitives " ou la dimension rebelle du connaître en régime de passion, Revue d'anthropologie des connaissances, 3, pp. 369-385.

Salzbrunn Monika (2017) Musique, religion, appartenances multiples : une approche de l'événement, Sociétés plurielles, 1, [en ligne]. URL : https://societes-plurielles.episciences.org/3668/pdf

Sawyer Lena (2006) Racialization, gender, and the negotiation of power in Stockholm's African dance courses, in Kamari Maxine Clarke and खDeborah A. Thomas Eds., Globalization and Race:Transformations in the Cultural Production of Blackness, Durham, Duke University Press, pp. 316-334.

Seye Elina (2014) Performative Participation: Embodiment of Identities and Relationships in Dance Events, in Linda Dankworth and Ann David Eds., Dance Ethnography and Global Perspectives, London, Palgrave Macmillan, pp. 58-74.

Sieveking Nadine (2002) Empowerment via embodiment: The transcultural practice of African dance in Berlin, Sociologus, 52 (2), pp. 215-244.

Tang Patricia (2007) Masters of the Sabar: Wolof Griot Percussionists of Senegal, Philadelphia, Temple University Press, 209 p.

Tholon Marie Sophie (2009) Du sable à la scène : circulation des danses sabar et ballet manding au Sénégal, entre gueew et ballet, Thèse de doctorat, Université de Nice.

Turino Thomas and James Lea (Ed.) (2004) Identity and the Arts in Diaspora Communities, Warren, Harmonie Park Press, 144 p. 


\section{Alice Aterianus-Owanga}

\section{"Sabar, sama thiosanou " (sabar, ma tradition). Frontières et propriété culturelles dans la transmission des danses sénégalaises en Europe}

Cet article interroge les jeux de frontières et d'appartenances en œuvre dans la transmission de la danse sénégalaise sabar en Europe. Après avoir retracé la construction du sabar comme répertoire national au Sénégal, l'article se penche sur les modalités d'implantation de cette performance en France et en Suisse. L'ethnographie du réseau européen du sabar éclaire d'abord la façon dont la mise en place de pédagogies spécifiques a permis de conférer une dimension " universelle " au sabar, tout en maintenant l'affirmation d'identifications nationales, par des circulations et des conversions des élèves qui s'intéressent à cette performance. Dans un second temps, I'approche des polémiques sous-jacentes à la transmission du sabar révèle que par-delà les inclusions partielles, les situations d'interaction et de rencontre ne dissolvent pas l'existence de frontières, dont la réaffirmation permet aux artistes de sabar de contester les inégalités économiques persistant entre les acteurs de ce champ.

\section{"Sabar, sama thiosanou" (Sabar, my Tradition). Cultural Boundaries and Property in the Transmission of Senegalese Dances in Europe}

This essay deals with the play on boundaries and belongings which are at stake through the transmission of Senegalese dance sabar in Europe. After having recounted the construction of sabar as a national repertoire in Senegal, it examines the implantation of this performance in France and Switzerland. The ethnography of sabar network highlights first how the development of a specific pedagogy has allowed to confer a universal dimension to this practice, while maintaining the assertion of national identifications, through circulations and conversions of sabar students. In a second part, the analysis of polemics underlying the transmission of sabar reveals that beyond inclusion, situations of interaction and encounter don't dissolve boundaries, which are reasserted by sabar artists in order to contest some economic inequalities persisting between the actors of this field.

\section{"Sabar, sama thiosanou» (sabar, mi tradición). Fronteras y propiedad culturales en la transmisión de los bailes senegaleses en Europa}

Este artículo cuestiona los juegos fronterizos y las afiliaciones en la transmisión de la danza senegalesa "Sabar» en Europa. Después de haber descrito la construcción del Sabar como repertorio nacional en Senegal, el artículo analiza cómo se implementa su actuación en Francia y Suiza. La etnografía de la red europea de Sabar primero ilumina la forma en que la implementación de pedagogías específicas ha permitido conferir una dimensión "universal" al Sabar, manteniendo al mismo tiempo la afirmación de las identificaciones nacionales, mediante circulaciones y conversiones mujeres interesadas en su actuación. En segundo lugar, el enfoque de las polémicas subyacentes a la transmisión del Sabar revela que, más allá de las inclusiones parciales, las situaciones de interacción y encuentro no disuelven la existencia de fronteras, cuya reafirmación permite a los artistas Sabar desafiar las desigualdades económicas persistentes entre los actores en este campo. 